02

\title{
Исследование режимов смешения туннельных сверхпроводниковых структур
}

\author{
(C) К.В. Калашников, А.А. Артанов, Л.В. Филиппенко, В.П. Кошелец \\ Институт радиофизики и электроники им. В.А. Котельникова РАН, \\ Москва, Россия \\ E-mail: kalashnikov@hitech.cplire.ru
}

\begin{abstract}
Экспериментально исследованы режимы работы туннельного перехода сверхпроводник-изоляторсверхпроводник (СИС) на основе трехслойных структур $\mathrm{Nb} / \mathrm{AlO}_{x} / \mathrm{Nb}$ и $\mathrm{Nb} / \mathrm{AlN} / \mathrm{NbN}$ в качестве гармонического смесителя (для частот порядка 600 и $20 \mathrm{GHz}$ ) и преобразователя частоты вверх (в диапазоне 0.1-5 GHz). Проведено сравнение квазичастичного и джозефсоновского режимов смешения. Показано, что в некоторых приложениях, таких как использование СИС-перехода в качестве криогенного гармонического фазового детектора, джозефсоновский режим может быть более предпочтительным, поскольку позволяет реализовать как больший сигнал, так и большее соотношение сигнал/шум по сравнению с квазичастичным. Также показана перспективность использования джозефсоновского режима смешения в системах мультиплексирования сигналов для сверхпроводниковых детекторов.
\end{abstract}

Работа выполнена при финансовой поддержке Минобрнауки РФ (соглашение № 14.613.21.0046, идентификатор RFMEFI61315X0046).

Смесительные сверхпроводниковые наноструктуры на основе туннельных переходов широко используются в приемных системах терагерцевого диапазона, поскольку обладают чрезвычайно высокой нелинейностью и предельно низкими собственными шумами [1-3]. Известно, что смесительные свойства контактов сверхпроводникизолятор-сверхпроводник (СИС) обусловлены двумя типами нелинейности: квазичастичным и джозефсоновским. Первый из них связан с туннельным током квазичастичных возбуждений из одного электрода перехода в другой, значительно возрастающим при приложении к переходу „щелевого“ напряжения, равного $V_{g}=\left(\Delta_{1}+\Delta_{2}\right) / e$, где $\Delta_{1,2}-$ величины энергетической щели первого и второго сверхпроводника, $e-$ заряд квазичастицы, равный заряду электрона. Второй тип нелинейности связан с бездиссипативным током куперовских пар, описываемым первым соотношением Джозефсона $I_{s}=I_{c} \sin \varphi$, где $\varphi-$ разность фаз сверхпроводящих берегов, $I_{c}$ - критический ток перехода.

Известны теоретические [4,5] и экспериментальные [6,7] работы по исследованию шумовых свойств смесителей на основе СИС-переходов, работающих в джозефсоновском режиме. В данных работах показано, что наличие неподавленного критического тока приводит к увеличению уровня шумов, что обычно связывают как с нестабильностью рабочей точки, так и с наличием собственных шумов джозефсоновской компоненты. В практических гетеродинных СИС-приемниках терагерцевого диапазона эффект Джозефсона традиционно считается паразитным, в связи с чем критический ток СИС-перехода, играющего роль смесительного элемента, обычно подавляется внешним магнитным полем $[8,9]$. Отметим, что во всех перечисленных публикациях работа СИС-смесителя изучена в режиме малой мощности гетеродина, частота которого совпадает с частотой сигнала, тогда как подробного экспериментального иссле- дования джозефсоновского режима для гармонических смесителей (в случае большой мощности гетеродина) до последнего времени опубликовано не было. В настоящей работе сделана попытка восполнить этот пробел и показано, что в некоторых практических приложениях джозефсоновское смешение является предпочтительным режимом работы.

Одним из новых применений туннельного СИСперехода является его использование в качестве криогенного гармонического фазового детектора (КГФД) для сверхширокополосных систем стабилизации излучения криогенных терагерцевых генераторов [10,11]; было показано, что функциональное объединение гармонического смесителя (ГС) и фазового детектора в одном элементе позволяет значительно расширить полосу синхронизации системы фазовой автоподстройки частоты (ФАПЧ) и улучшить спектральное качество.

Поскольку качество синхронизации напрямую зависит от амплитуды выходного сигнала КГФД, необходимы оптимизация режимов его работы и исследование режимов смешения в ГС. Экспериментальная установка для изучения характеристик ГС представлена на рис. 1 и включает в себя туннельный СИС-переход на основе трехслойной структуры $\mathrm{Nb} / \mathrm{AlO}_{x} / \mathrm{Nb}$, интегрированный на микросхеме с планарной дипольной антенной (рассчитанной на частотный диапазон 450-700 GHz) и соединенный с направленным ответвителем и устройством задания постоянного смещения (англ. bias tee), размещенными в заливном гелиевом криостате при температуре $4.2 \mathrm{~K}$. На СИС-переход подаются два сигнала: антенна принимает сигнал частотой около $500 \mathrm{GHz}$ (умноженный сигнал лампы обратной волны диапазона $120-160 \mathrm{GHz}$, стабилизированный системой ФАПЧ), а через направленный ответвитель на образец подается сигнал опорного синтезатора частоты около $20 \mathrm{GHz}$, конкретное значение частоты подбирается таким обра- 


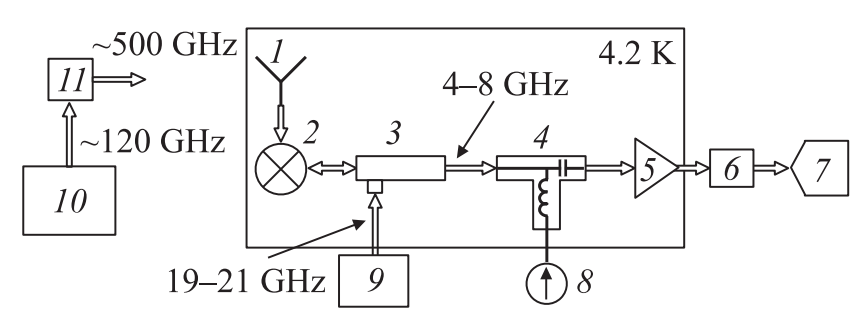

Рис. 1. Схема экспериментальной установки измерения характеристик ГС на СИС-переходе. 1 - антенна, $2-$ ГС, 3 - направленный ответвитель, 4 - ответвитель для задания смещения СИС-перехода, 5 - система усилителей, 6 - полосовой фильтр, 7 - измеритель мощности, 8 - источник тока, позволяющий задавать напряжение смещения на СИСпереходе, 9 - опорный синтезатор, 10 - лампа обратной волны, 11 - сверхрешеточный умножитель.
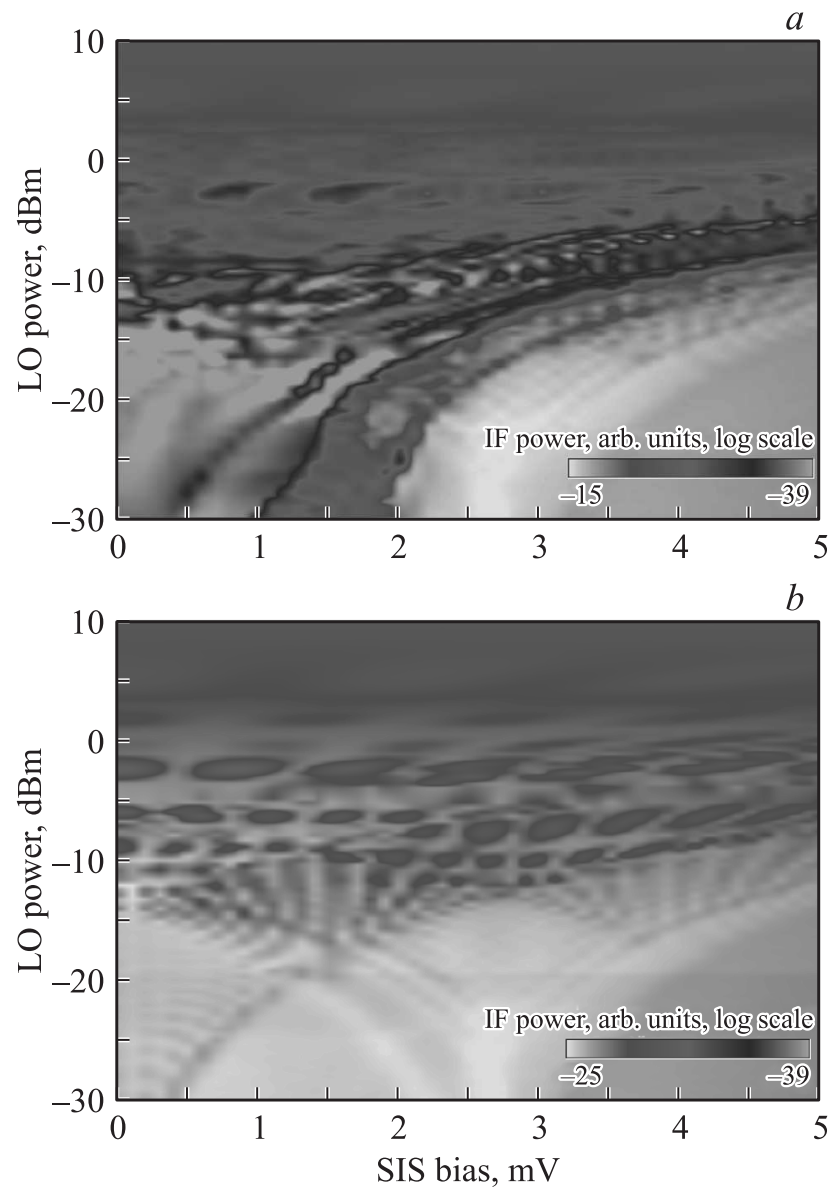

Рис. 2. Зависимость мощности сигнала в тракте ПЧ от напряжения смещения на СИС-переходе и мощности сигнала опорного синтезатора для джозефсоновского $(a)$ и квазичастичного $(b)$ смешения.

зом, чтобы сигнал промежуточной частоты (ПЧ) попадал в полосу каскада усилителей 4-8 GHz. С помощью тока контрольной линии, расположенной на одном чипе с СИС-смесителем, возможно создание магнитного поля на образце и подавление критического тока СИСперехода.
Сигнал ПЧ после прохождения узкополосного перестраиваемого ЖИГ-фильтра (ЖИГ - железо-иттриевый гранат, ширина полосы $70 \mathrm{MHz}$ ) регистрировался с помощью спектроанализатора или измерителя мощности. Для исследования режимов работы ГС удобно воспользоваться зависимостью мощности выходного ПЧсигнала от постоянного напряжения смещения на ГС и мощности сигнала синтезатора. На рис. 2 приведены две такие зависимости для случаев полностью подавленного (квазичастичный режим смешения) и неподавленного (джозефсоновский режим) критического тока СИСперехода.

Из рисунка видно, что изучаемая зависимость в квазичастичном режиме смешения имеет сложный характер расходящихся пиков [12,13], при этом измеряемая в тракте ПЧ мощность значительно увеличивается в режиме без подавления джозефсоновского тока магнитным полем. Более подробное изучение сигнала ПЧ показало, что в джозефсоновском режиме смешения происходит также существенное увеличение уровня шумовой подставки. Измерение отношения сигнал/шум для исследуемых режимов проводилось следующим образом: сначала измерялась мощность в тракте ПЧ в случае попадания сигнала ПЧ в полосу ЖИГ-фильтра, затем частота внешнего синтезатора незначительно изменялась так, чтобы вывести сигнал ПЧ из полосы фильтра, при этом измеритель мощности регистрировал только шумовую мощность. Отметим, что данное измерение следует проводить при подаче на СИС-смеситель сигнала гетеродина, поскольку его отключение изменяет шумовую подставку. Как видно из данных, представленных на рис. 3, для джозефсоновского режима смешения существуют оптимальные значения приложенной мощности опорного синтезатора. При мощности меньше $-12 \mathrm{dBm}$ происходит резкое возрастание уровня шума (более чем на $15 \mathrm{~dB}$ ) в джозефсоновском режиме с малым отношением сигнал/шум (около $3 \mathrm{~dB}$ ), при увеличении мощности опорного синтезатора выше $5 \mathrm{dBm}$ эффект Джозефсона подавляется приложенным сигналом и смешение осуществляется только на квазичастичной нелинейности.

Для джозефсоновского режима преобразования реализуется примерно на $4 \mathrm{~dB}$ большее соотношение сигнал/шум, чем при подавленном критическом токе, что может быть связано с частичной синхронизацией джозефсоновской компоненты тока мощным сигналом опорного синтезатора. Такой эффект не реализуется в классических смесителях с маломощным и высокочастотным сигналом гетеродина. Большая эффективность джозефсоновского преобразования приводит к тому, что использование СИС-смесителя в качестве КГФД для системы ФАПЧ для стабилизации линии излучения генератора на основе длинного джозефсоновского перехода (ДДП) шириной $17.9 \mathrm{MHz}$ позволяет синхронизировать до $83 \%$ мощности, тогда как подавление эффекта Джозефсона в КГФД уменьшает эту величину до $70 \%$ (рис. 4). Таким образом, джозефсоновский режим является более 

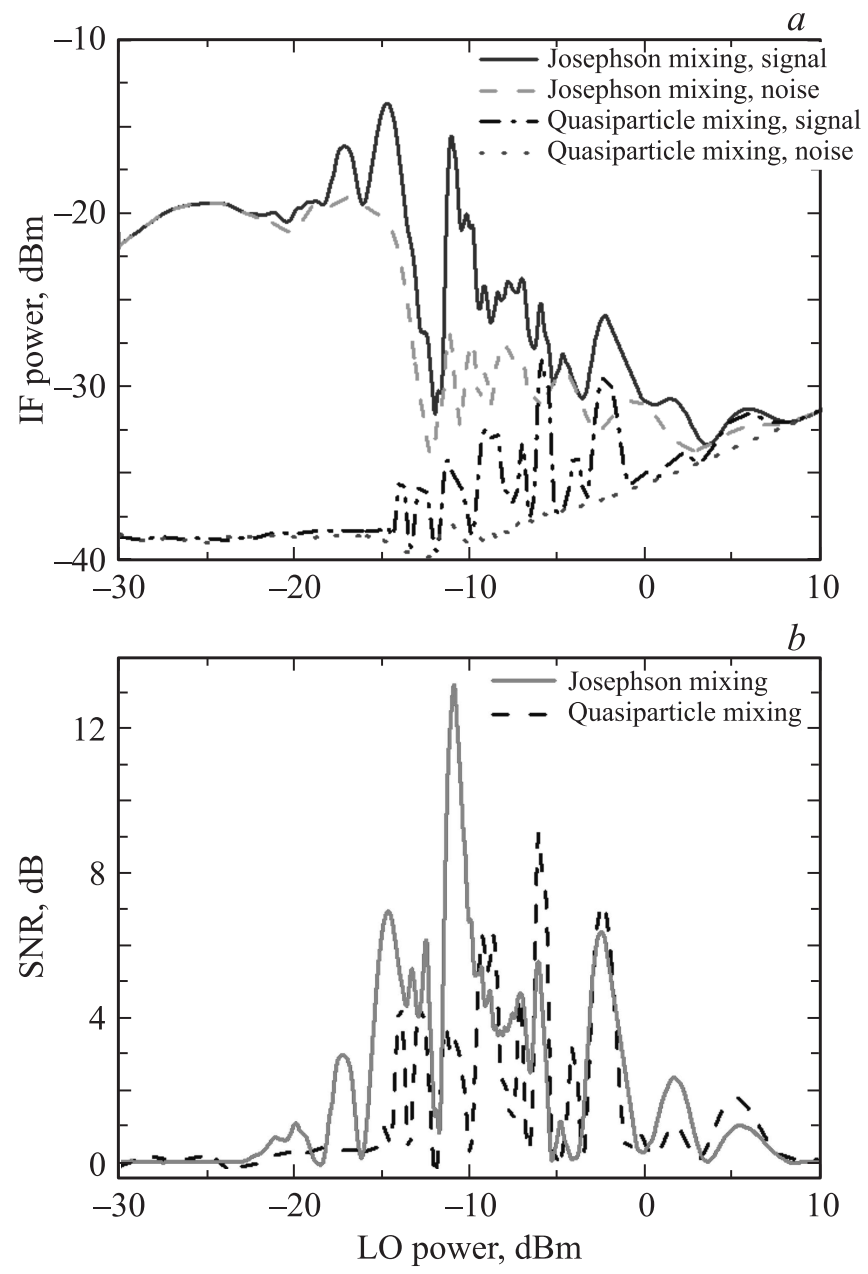

Рис. 3. Экспериментальные зависимости мощностей сигнала ПЧ и шумовой подставки $(a)$ и отношение сигнал/шум $(b)$ от мощности сигнала опорного синтезатора при нулевом напряжении смещения для различных режимов смешения.

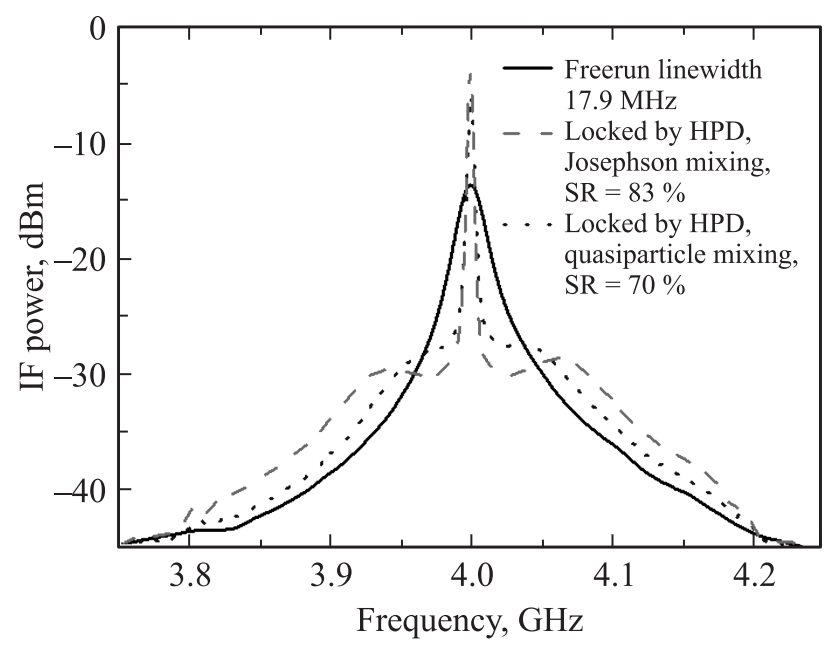

Рис. 4. Пониженные по частоте спектры генерации ДДП: автономная линия (сплошная кривая), фазовая синхронизация с помощью КГФД в квазичастичном (пунктирная кривая) и джозефсоновском (штриховая кривая) режимах.

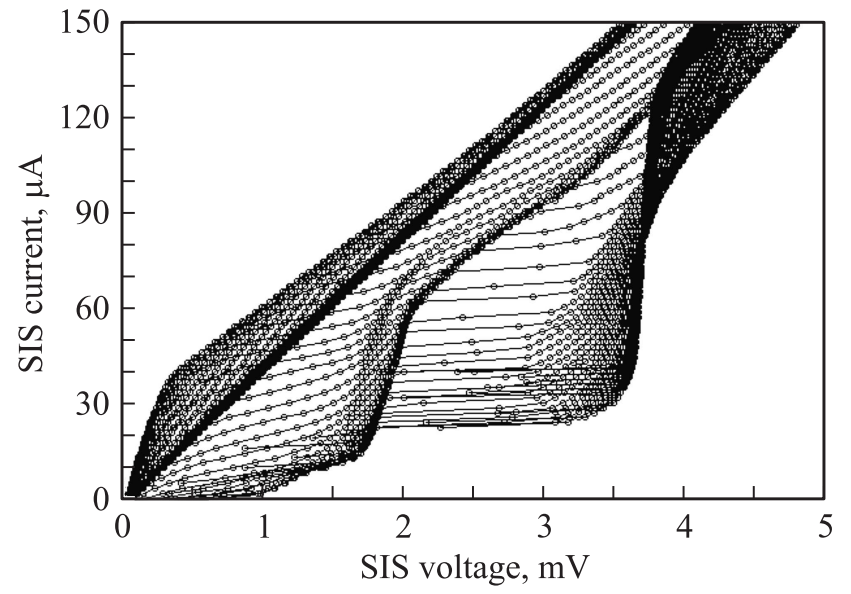

Рис. 5. Семейство вольт-амперных характеристик СИСперехода с неподавленным критическим током под воздействием сигнала частотой $5 \mathrm{GHz}$ разной мощности в диапазоне от -50 до $-20 \mathrm{dBm}$.

предпочтительным для эффективного функционирования СИС-перехода в качестве КГФД.

Еще одним перспективным применением смесителей на основе туннельных СИС-переходов является их использование в системах мультиплексирования для считывания сигнала с многопиксельных приемников, основанных на болометрах на краю сверхпроводящего перехода [14]. Для изучения возможности использования СИС-перехода в качестве преобразователя вверх по частоте была модифицирована экспериментальная установка, показанная на рис. 1: теперь через направленный ответвитель на СИС-переход могли подаваться два СВЧсигнала одновременно. С помощью модифицированной установки исследовались потери преобразования сигнала частотой $223 \mathrm{MHz}$ в сигнал $5.223 \mathrm{GHz}$ с помощью гетеродина $5 \mathrm{GHz}$ в зависимости от параметров подаваемых сигналов. Совместно с измерением трехмерных зависимостей мощности ПЧ сигнала от напряжения смещения и мощности сигнала гетеродина были получены семейства вольт-амперных характеристик СИС-перехода на основе трехслойной структуры $\mathrm{Nb} / \mathrm{AlN} / \mathrm{NbN}$ для различных значений приложенной мощности (рис. 5).

В представленном семействе ВАХ четко видна особенность, связанная с резким повышением тока при некоторых напряжениях диапазона $2-3 \mathrm{mV}$ с последующим скачком в область напряжений, близких к щелевому. Исследования показали, что данная особенность, исчезающая при подавлении критического тока перехода, не связана с резонансной структурой, в которую включен СИС-переход, и имеет природу, близкую к эффекту самонакачки в ДДП [15]. Поскольку при скачке напряжения реализуется большое значение дифференциального сопротивления СИС-перехода, в данных рабочих точках следует ожидать высокого коэффициента преобразования смесителя.

На рис. 6 представлены зависимости потерь преобразования от напряжения смещения на переходе для 


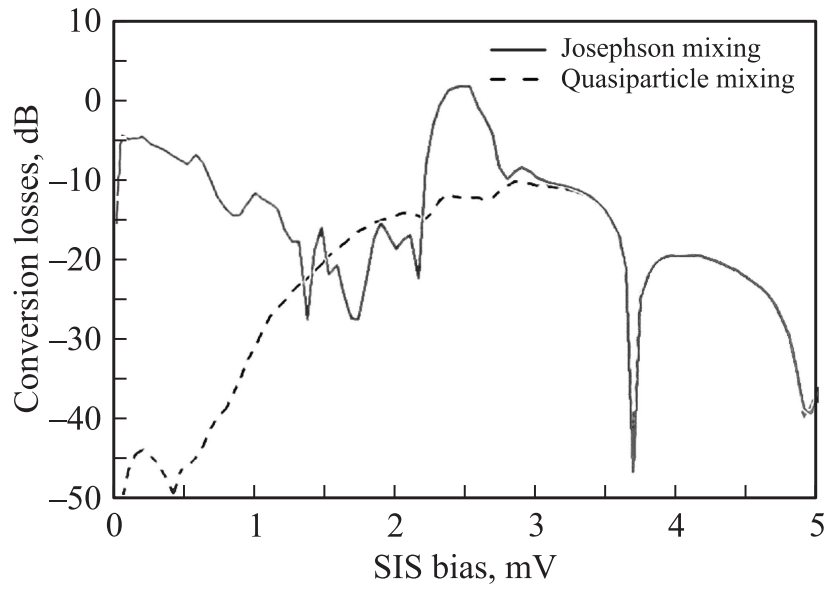

Рис. 6. Потери преобразования вверх по частоте сигнала 223 МГц в зависимости от напряжения смещения на СИСпереходе для джозефсоновского (сплошная кривая) и квазичастичного (штриховая кривая) смешения.

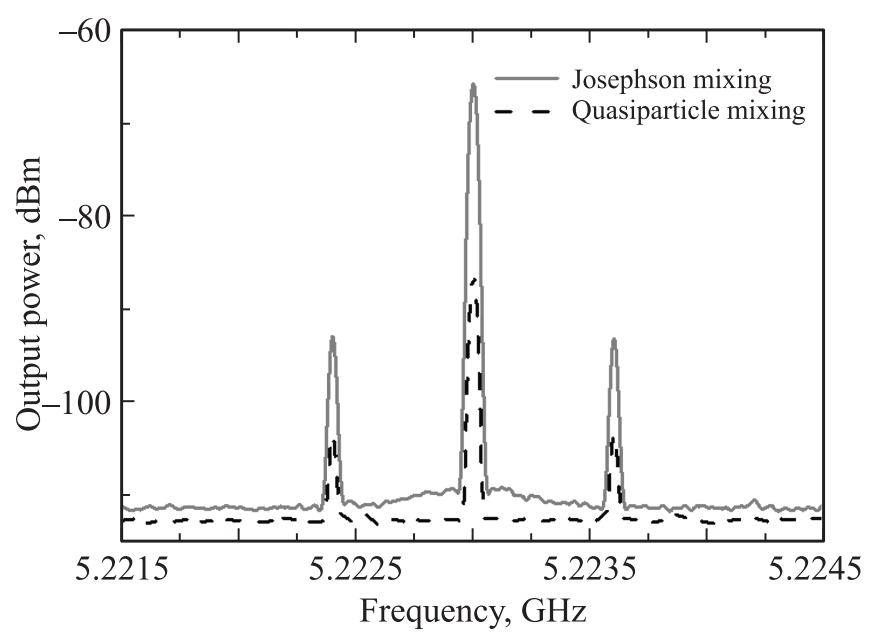

Pис. 7. Спектры выходного сигнала преобразователя при напряжении $2.6 \mathrm{mV}$ для джозефсоновского (сплошная кривая) и квазичастичного (штриховая кривая) смешения.

мощности гетеродина $-40 \mathrm{dBm}$, из которых следует, что оптимальным напряжением при данном уровне накачки является $2.5 \mathrm{mV}$. Из рис. 7, на котором показаны спектры преобразованных вверх по частоте сигналов в рассматриваемой рабочей точке, следует, что джозефсоновский режим позволяет реализовать более высокую мощность при малом увеличении шумов по сравнению с квазичастичным.

Как видно из данного рисунка, в джозефсоновском режиме смешения в обсуждаемом диапазоне напряжений реализуются значительно более низкие потери преобразования и более высокое соотношение сигнал/шум, чем в квазичастичном, что делает данный режим работы более предпочтительным при работе со слабыми сигналами. С другой стороны, в квазичастичном режиме реализуется более плавная зависимость потерь преобразования от смещения, что существенно при выборе рабочей точки смесителя.

Таким образом, в работе показано, что в некоторых практических приложениях смесителей на основе туннельных СИС-переходов желательно наличие не только квазичастичной, но и джозефсоновской нелинейности. При работе СИС-перехода в режиме гармонического смесителя существует оптимальная мощность опорного синтезатора, позволяющая не только значительно увеличить мощность выходного сигнала, но и существенно уменьшить джозефсоновские шумы. При оптимальных величинах мощности гетеродина джозефсоновский режим позволяет реализовать как высокое значение выходного сигнала (по сравнению с характерным для квазичастичного режима), так и большее значение отношения сигнал/шум. Джозефсоновский режим также позволяет получить более низкие потери преобразования при использовании СИС-перехода в качестве мультиплексора для сигналов порядка $0.5-5 \mathrm{GHz}$.

\section{Список литературы}

[1] A.B. Zorin. IEEE Trans. Magn. 21, 939 (1985).

[2] J.R. Tucker, M.J. Feldman. Rev. Mod. Phys. 4, 1055 (1985).

[3] A. Karpov, J. Blondell, M. Voss, K.H. Gundlach. IEEE Trans. Appl. Supercond. 5, 2, 3304 (1995).

[4] B.A. Huberman, J.P. Crutchfield, N.H. Packard. Appl. Phys. Lett. 37, 8, 750 (1980).

[5] N.F. Pedersen, A. Davidson. Appl. Phys. Lett., 39, 10, 830 (1981).

[6] M.J. Wengler, N.B. Dubash, G. Pance, R.E. Miller. IEEE Trans. Microwave Theory Techn. 40, 5, 820 (1992).

[7] B. Leridon, P. Febvre, S. George, P. Feautrier, W.R. McGrath. J. Appl. Phys. 82, 6, 3024 (1997).

[8] J. Zmuidzinas, J.W. Kooi, J. Kawamura, G. Chattopadhyay, B. Bumble, H.G. LeDuc, J.A. Stern. Proc. SPIE 3357, 53 (1998).

[9] T.G. Phillips, D.P. Woody, G.J. Dolan, R.E. Miller, R.A. Linke. IEEE Trans. Magn. 17, 1, 684 (1981).

[10] K.V. Kalashnikov, A.V. Khudchenko, V.P. Koshelets. Appl. Phys. Lett. 103, 102601 (2013).

[11] K.V. Kalashnikov, A.V. Khudchenko, V.P. Koshelets. In: 25th Int. Symp. on space terahertz technology (ISSTT-2014). M. (2014). P. 85.

[12] К.В. Калашников, А.В. Худченко, А.М. Барышев, В.П. Кошелец. Радиотехника и электроника 56, 6, 751 (2011).

[13] S. Withington, P. Kittara, G. Yassin. J. Appl. Phys. 93, 12, 9812 (2003).

[14] G. de Lange. J. Low Temp. Phys. 176, 3-4, 408 (2014).

[15] V.P. Koshelets, S.V. Shitov, A.V. Shchukin, L.V. Filippenko, J. Mygind, A.V. Ustinov. Phys. Rev. B 56, 5572 (1997). 